\title{
Molecular Imaging to Monitor Left Ventricular Remodeling in Heart Failure
}

\author{
Elias Ylä-Herttuala ${ }^{1}$ • Antti Saraste ${ }^{2,3} \cdot$ Juhani Knuuti ${ }^{2,3} \cdot$ Timo Liimatainen $^{4,5} \cdot$ Seppo Ylä-Herttuala ${ }^{1,6}$
}

Published online: 26 February 2019

(C) The Author(s) 2019

\begin{abstract}
Purpose of Review Cardiovascular diseases are the leading cause of deaths worldwide. Many complex cellular and molecular pathways lead to myocardial remodeling after ischemic insults. Anatomy, function, and viability of the myocardium can be assessed by modern medical imaging techniques by both visualizing and quantifying damages. Novel imaging techniques aim for a precise and accurate visualization of the myocardium and for the detection of alternations at the molecular level.

Recent Findings Magnetic resonance imaging assesses anatomy, function, and tissue characterization of the myocardium noninvasively with high spatial resolution, sensitivity, and specificity. Using hyperpolarized magnetic resonance imaging, molecular and metabolic conditions can be assessed non-invasively. Single photon-emission tomography and positron-emission tomography are the most sensitive techniques to detect biological processes in the myocardium. Cardiac perfusion, metabolism, and viability are the most common clinical targets. In addition, molecular-targeted imaging of biological processes involved in heart failure, such as myocardial innervation, inflammation, and extracellular matrix remodeling, is feasible.

Summary Novel imaging techniques can provide a precise and accurate visualization of the myocardium and for the detection of alternations at molecular level.
\end{abstract}

Keywords Myocardium · Infarction · MRI · Hyperpolarized MRI $\cdot$ PET · SPECT

\section{Introduction}

Cardiovascular diseases (CVD) are the leading cause of death worldwide and consist of a variety of diseases from cardiac dysfunction to aneurysm rupture [1-3]. In this review, we describe how novel imaging techniques including magnetic resonance imaging (MRI), hyperpolarized MRI (hMRI), positron-emission tomography (PET), and single photon-emission computed tomography (SPECT) techniques can be used to image myocardial infarction (MI) and myocardial remodeling post-MI. MI develops when reduced blood flow and lack of oxygen lead to death of cardiomyocytes via necrosis, inflammation, replacement of extracellular space by fibrosis, protein infiltration, and myocardial disarray $[4,5]$. Overall, fibrosis is thought to be the final common pathway in various myocardial

This article is part of the Topical Collection on Molecular Imaging

Seppo Ylä-Herttuala

Seppo.Ylaherttuala@uef.fi

Elias Ylä-Herttuala

elias.yla-herttuala@uef.fi

Antti Saraste

antti.saraste@utu.fi

Juhani Knuuti

juhani.knuuti@utu.fi

Timo Liimatainen

timo.liimatainen@oulu.fi
1 A.I. Virtanen Institute for Molecular Sciences, University of Eastern Finland, P.O. Box 1627, FI-70211 Kuopio, Finland

2 Turku PET Centre, Turku University Hospital and University of Turku, Turku, Finland

3 Heart Center, Turku University Hospital, Turku, Finland

4 Research Unit of Medical Imaging, Physics and Technology, University of Oulu, Oulu, Finland

5 Department of Diagnostic Radiology, Oulu University Hospital, Oulu, Finland

6 Heart Center, Kuopio University Hospital, Kuopio, Finland 
diseases [6]. Intense fibrosis leads to scar formation [7••, 8-11], which further changes the shape and function of the left ventricle (LV) and can be manifested as dilation and thinning of the myocardium, hypertrophy of the remote areas surrounding MI, and overall decline of the heart function (Fig. 1) [3, 12-14]. All of these features can be accessed by kinematic imaging over the heart cycle [3].

To image heart function, ultrasound and computed tomography $(\mathrm{CT})$ are applied besides the above mentioned techniques $[3,15,16]$. Nuclear medicine imaging techniques, namely PET and SPECT, are used to measure changes in perfusion, metabolism, and molecular pathways at the cellular level during the remodeling [3]. Tracer molecule owing a capability to bind into defined receptor or other molecule steers radionuclide or other compound which enhances image contrast into target tissue [17]. Although tracers have been developed for MRI and SPECT, the most sensitive technique is still PET [3]. To add sensitivity of MRI, novel techniques based on hMRI have been developed which have enabled imaging of the cell cycle and cell metabolism [18•].

\section{MRI}

Cardiac magnetic resonance imaging (cMRI) contains a wide variety of options to study cardiac anatomy, function, infarct scar, and fibrosis and to characterize the myocardium by relaxation time mapping, perfusion, water diffusion, wall movement, and myocardial stiffness $[19,20]$. cMRI is currently the golden standard to assess anatomy and function of the myocardium non-invasively with high spatial resolution and accuracy $[3,15,16]$.

\section{Functional cMRI}

The anatomy and functional images from the LV are typically imaged by acquiring rapidly either with multiple 2D- or 3Dcine images that are covering the whole heart during more than $95 \%$ of the cardiac cycle [21]. Images allow the accurate determination of end diastolic volume (EDV) and end systolic volume (ESV) which are further used to calculate ejection fraction (EF), stroke volume (SV), and cardiac output (CO) $[3,12,15,16,22]$. Reduced EF together with increased EDV and decreased myocardial thickness are the clearest signs of reduced systolic function and remodeling [12, 13, 15, 23, 24]. Additionally, EF and EDV have been shown to increase as a function of time after MI reperfusion in human [25], swine [20], and mice [26]. As LV becomes globally thinner, MI scar tissue expands and causes extra workload in the LV, hypertrophy, and expansion of the healthy myocytes to maintain $\mathrm{CO}$ $[12,27]$. Recently, texture analysis applied on MRI cine images was demonstrated to differentiate nonviable and viable MI areas and remote areas of the myocardium [28]. Texture analysis finds the patterns and relationships among pixels from heterogeneities within the imaging target [28].

There are also several other methods to study anatomy and function of the heart. Bright-blood technique is used for the detection of hemorrhage in the myocardium [29] whereas dark-blood technique is used to improve the discrimination of myocardium and adjacent blood pool revealing small areas of MI in the endocardium [30]. Myocardial tagging is an imaging technique which assesses the motion and deformation of LV myocardium with good temporal and spatial resolution [31]. Therefore, myocardial deformation and motion stiffness caused by MI and fibrosis can be detected by myocardial
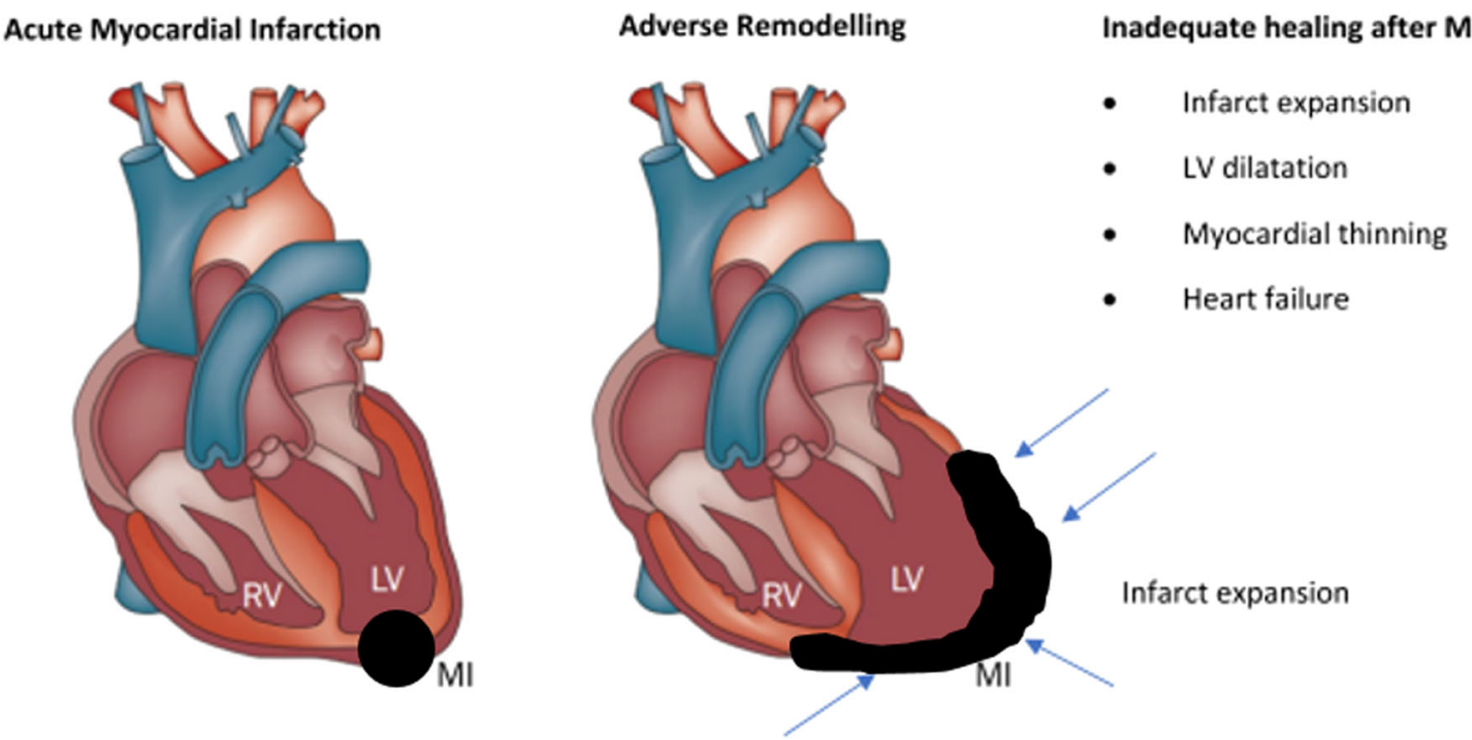

Fig. 1 Schematic picture showing gross changes in adverse cardiac remodeling post-MI (adapted with permission from Springer Nature: van den Borne SW) [14] 
tagging [32, 33]. Feature tracking is a novel image analysis technique for myocardial tagging that was shown to be a feasible and robust technique to detect LV motion where data analysis is faster compared to myocardial tagging [33].

\section{Contrast Agent Imaging: Late Gadolinium Enhancement and Extracellular Volume}

The current gold standard to assess and localize the chronic MI area in clinics is the contrast agent (CA)-based late gadolinium enhancement (LGE) technique. Gadolinium (Gd) accumulates into expanded extracellular space in irreversibly damaged MI tissue and its washout back to the blood stream is delayed, which shortens $T_{1}$ relaxation time in the MI area [7••]. Thus, Gd accumulation is detected as a delayed hyperintensity in $T_{1^{-}}$ weighted MR images (Fig. 2) [7••]. Delayed hyperintensity in the myocardium and signal from blood is hard to distinguish. To improve contrast between infarct scar and blood in LGE, a $T_{2}$ preparation was added between inversion pulse and image acquisition [34]. Cons of the LGE technique include the inability to show the quality of the scar, emphasis on extracellular water content, and failure to detect diffuse fibrosis and global changes in the myocardium after the injury $[15,35]$. In a recent myocardial permeability study, measured with albumin-bound $\mathrm{Gd}$, alternations were associated with remodeling between acute and chronic MI [36]. $T_{1}$ mapping before and after contrast injection together with hematocrit were essential to calculate the extracellular volume (ECV) $[15,16,37]$. Larger ECV fractions were measured in MI $(54 \pm 1 \%)$ than in remote myocardial tissue $(29 \pm 2 \%)$ [38]. Similar ECV differences between MI and healthy myocardium $(25 \pm 3 \%)$ have been reported in the myocardium [39].

ECV technique is a sensitive method to detect the distribution of the cellular and extracellular interstitial matrix compartments [6]. It has been shown to reflect the extent of myocardial fibrosis and has been validated against collagen volume fraction (Table 1) [40]. It has been reported to agree better with the collagen volume fraction than the post-contrast $T_{1}$ alone [41] and to be more accurate in the detection of acute MI compared to LGE [7••]. Additionally, ECV is used to evaluate the transmural extent of MI $[7 \bullet \bullet]$.

\section{Conventional Relaxation Times}

Visualization of the myocardial tissue and detection of both acute and chronic MI can be done without CA. These techniques offer quantitative assessment of the alternations in the composition of myocardial tissue based on intrinsic water properties, longitudinal $T_{1}[7 \bullet \bullet, 42,43]$ and transversal $T_{2}$ relaxations $[42,44]$, to generate contrast within the myocardium [6]. $T_{1}$-weighted images are typically used for anatomical imaging and $T_{2}$-weighted images for edema and imaging of transient ischemia (Table 1) [45]. $T_{1}$ and $T_{2}$ relaxations can be mapped by acquiring multiple relaxation weighted images and by fitting a curve to signal intensities pixel-by-pixel manner to form relaxation time maps $[35,44,46]$. Both $T_{1}$ and $T_{2}$ mapping also allow visualization and quantification of global changes in the myocardium (Table 1) [11, 44, 46].

$T_{1}$ relaxation time is elevated during MI development [6, $10,38,39,47]$ and it has been shown to distinguish between reversible and irreversible damages in post ST-elevation MI (STEMI) [22, 48, 49]. In chronic $\mathrm{MI}$, native $T_{1}$ relaxation times are lower than in acute MI because edematous and necrotic tissues in the acute MI are replaced by smaller amounts of expanded extracellular collagen $[7 \bullet \bullet, 50]$. Therefore, $T_{1}$ mapping can be used for diagnostic purposes to detect different pathological states in the myocardium (Table 1) $[6,39$, 40].

$T_{2}$ relaxation determines edema via increased $T_{2}$ relaxation time resulting from an increased amount of interstitial free water [28]. Therefore, $T_{2}$ relaxation time is suitable for the determination of the area at risk in acute MI [6, 28, 40]. However, $T_{2}$ suffers from a poor contrast-to-noise ratio compared to $T_{1}$ and longitudinal rotating frame relaxation time $\left(T_{1 \rho}\right)$ [51]. $T_{2} *$ relaxation time can be chosen when myocardial iron content is used to detect myocardial hemorrhage since $T_{2}$ * relaxation time is more sensitive for magnetic susceptibilities than $T_{2}$ due to the accumulated iron content (Table 1) [15, $28,40,52]$.

\section{$T_{1 \rho}$ Relaxation Time}

Another advanced technique is based on the mapping of a longitudinal rotating frame relaxation time $\left(T_{1 \rho}\right)$ which measures relaxation during a radiofrequency $(\mathrm{RF})$ pulse $[42,53]$. $T_{1 \rho}$ relaxation is sensitive to slow molecular motions (range of 0.1 to $10 \mathrm{kHz}$ in vivo compared to fast molecular motions at Larmor frequency at 10-500 $\mathrm{MHz}$ which are used in $T_{1}$ and $T_{2}$ relaxation time measurements) [7••]. In general, $T_{1 \rho}$ relaxation time is always between $T_{1}$ and $T_{2}$ relaxation times approaching $T_{2}$ when spin-lock pulse power nears zero [51]. Increased $T_{1 \rho}$ relaxation time associates with increased extracellular volume and fibrotic area in $\mathrm{MI}[37,53]$ and correlates with LGE in mice (Fig. 2) [7••, 54], pigs [51], and humans [55] after MI. One limitation of the $T_{1 \rho}$ relaxation time mapping in clinics is the relatively high specific absorption rate (SAR) causing tissue heating $[7 \bullet \bullet]$.

\section{Relaxation Along Fictitious Field}

Relaxation along a fictitious field (RAFF) in the nth rotating frame (RAFFn) is a novel MRI relaxation time technique to perform rotating frame relaxation time measurements with

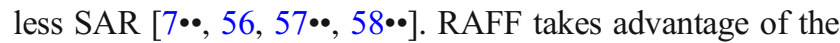
fictitious magnetic field which is produced by a fast sweep of the effective RF field [56, 57••]. Advantages of the low SAR 

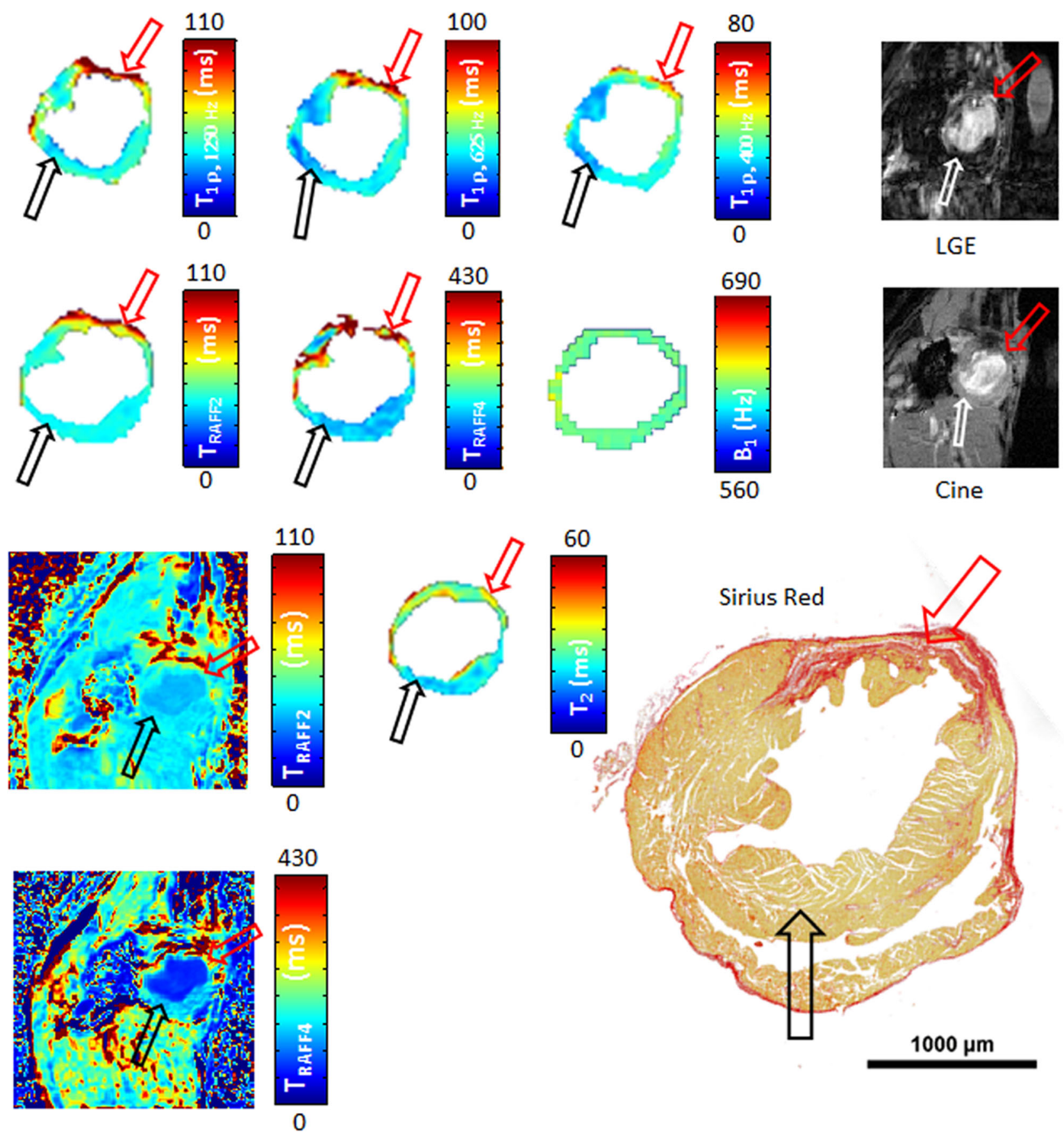

Fig. 2 Relaxation time maps, LGE, cine, and a corresponding histology image with sirius red-stained section from the infarcted mouse heart 21 days after infarct. Red arrow indicates the infarct area and black/

become more evident when RAFF in the higher rank (n) rotating frames (RAFFn), an extension of RAFF, is used. Typically, $n$ varies between 1 and 5 in imaging applications $[56,57 \bullet \bullet]$. The contrast between MI and remote areas has been demonstrated with RAFFn technique in mice and the MI area was equally accurately detected as with $T_{1 \rho}$, LGE, and histology (Fig. 2) [7••]. $T_{\text {RAFFn }}$ relaxation times are also elevated and more sensitive than $T_{1 \rho}$ in the detection of fibrotic area in the hypertrophic myocardium in mice [59].

white arrow shows the remote control area. B1 homogeneity was verified to be nominal $\pm 10 \% \mathrm{~Hz}$ in the area of the whole myocardium (adapted from Yla-Herttuala E) [7••]

\section{Hyperpolarization}

A novel imaging technique to measure real-time metabolic activity without ionizing radiation is hyperpolarized MRI (hMRI). The most common technique of hMRI is dynamic nuclear polarization (DNP). Most often, DNP is based on the dynamics of the downstream metabolism of $\left[1-{ }_{13} \mathrm{C}\right]$-labeled pyruvate which is the final product of the glycolytic glucose breakdown [18•]. In healthy myocytes and in aerobic 
Table 1 Feasibility of conventional parametric mapping methods in different diseases and tissue characteristics

\begin{tabular}{|c|c|c|c|c|c|}
\hline & & $T_{2}$ & $T_{2}{ }^{*}$ & $T_{1}$ & $\mathrm{ECV}$ \\
\hline \multirow[t]{3}{*}{ AMI } & Hemorrhage & • & $\bullet \bullet$ & • & $? ?$ \\
\hline & Edema & $\bullet$ & $? ?$ & $\bullet$ & • \\
\hline & Necrosis & • & $\bullet \bullet$ & $\bullet$ & $\bullet$ \\
\hline \multirow[t]{2}{*}{ Fibrosis } & Focal/regional* & $\circ$ & $\circ$ & - & $\bullet$ \\
\hline & Diffuse/global* & $? ?$ & $\circ$ & - & $\bullet$ \\
\hline
\end{tabular}

$\bullet=$ useable

$\cdot=$ potential

$? ?=$ unknown

${ }^{\circ}=$ not useable

*Diffuse/global refers to phenomena affecting to the whole myocardium and focal/regional refers to localized abnormalities in the myocardium

$A M I$, acute myocardial infarction; $E C V$, extracellular volume

conditions, pyruvate is converted to acetyl-CoA and $\mathrm{CO}_{2} /$ bicarbonate via pyruvate dehydrogenase $[18 \bullet]$. In anaerobic conditions, the lack of oxygen shifts energy conversion to lactate formation via lactate dehydrogenase [18•]. Imaging $\left[1{ }_{13} \mathrm{C}\right]-$ labeled pyruvate with hMRI is over 10,000 times more sensitive than the conventional MR spectroscopy which makes it possible to accurately characterize and image low natural abundances of metabolic compounds in healthy and ischemic tissues [18•]. hMRI with DNP has potential to add much more sensitivity and specificity for the characterization of the ischemic area and effects of the revascularization therapies since DNP technique reflects energy homeostasis [18 $]$. Fast $T_{1}$ decay $(\sim$ $45 \mathrm{~s}$ for $\left[1-{ }_{13} \mathrm{C}\right]$-labeled pyruvate) of the substrate limits the hMRI applications [18 ${ }^{\bullet}$. Big efforts have been made to make hMRI available for human use [60] although most hMRI cardiac imaging studies are still done in experimental animals [60-63]. In a pig reperfusion model, a significant increase in lactate level after myocardial reperfusion was found whereas bicarbonate level remained low after 5 min reperfusion [64] which clearly demonstrates fast metabolic alternations after reperfusion. Supporting these findings, an elevated level of lactate and a decreased level of bicarbonate were found in an ex vivo infarction study and myocardial reperfusion studies (Fig. 3) [61, 65, 66]. Moreover, in an in vivo porcine study, LV wall motion was retained when bicarbonate level returned back to normal, but LGE was unchanged after reperfusion (Fig. 3) [62]. Additionally, decreased $\mathrm{pH}$ due to increased glycolysis and intracellular proton and lactic acid production were found in ischemic myocardium $[18 \bullet, 67,68]$. Reduction of the Krebs cycle flux, where the ladder production from $\left[1-{ }_{13} \mathrm{C}\right]-$ pyruvate to different metabolic compounds takes place, was correlated to the LV systolic dysfunction in rats [69]. Along with the above myocardium studies, hMRI has been used to study diabetic cardiomyopathy, fibrosis, hypertrophy and coronary artery disease in animal models, and patients with promising results $[18 \bullet, 70]$.

\section{Other MRI Techniques}

Myocardial perfusion gives useful information about capillary blood flow in the myocardium. Myocardial perfusion can be measured without CA by techniques of arterial spin labeling [71] and blood oxygen level-dependent contrast [15]. CA is used in myocardial angiography, where blood flow inside coronary arteries can be measured since the blood flow is alternated in the area of MI compared to the surrounding heart muscle [72]. CAMRA has also been used to determine microvascular obstruction in swine acute MI model with high accuracy [22, 71]. Increased ECV, loss of cardiomyocytes and therefore loss of orientational structure, increases water diffusion in MI compared to the rest of the myocardium [3] which can be imaged with the diffusion weighted MRI [73]. Diffusion tractography, [3] measuring the orientation of cardiomyocytes, has grown in the CMRI field since diffusion tractography was introduced in rat MI model [74]; MI area is disturbing the normal form of crossing helical fiber architecture of normal myocytes. Bright-blood, which is gradient-echo based and black-blood, which is a spin-echo based, $T_{2}$-weighted sequence, can be used to assess myocardial structure, acute MI and ischemic areas with good accuracy in patients $[15,75]$. Stiffness of MI area and the rest of the myocardium is also studied by MRI elastography where the MI area is discriminated from the rest of the myocardium by the difference be-

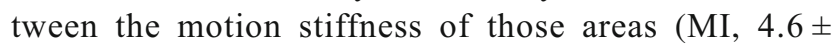
$0.7 \mathrm{kPA}$; healthy, $3.0 \pm 0.6 \mathrm{kPA})[73,76]$. Moreover, cMRI has an ability to determine with great accuracy mitral valve [77] and aortic valve [78] malfunctions, which often occur as MI develops and therefore imaging the function of mitral and aortic valves might give additional information about the heart's condition [77].

\section{SPECT and PET}

SPECT and PET represent nuclear imaging techniques that enable mapping of radiotracer concentration and kinetics in the myocardium with very high sensitivity [79]. Improvement in PET and SPECT imaging technology has led to the evolution of imaging beyond the isolated assessment of myocardial perfusion, toward molecular-targeted imaging of biological processes involved in heart failure, such as myocardial metabolism, innervation, inflammation, and extracellular matrix remodeling. PET and SPECT scanners are increasingly integrated with either CT or MRI systems into PET-CT or PET-MRI hybrid imaging devices, which facilitate the localization of a molecular signal, by fusion with high resolution morphologic images [80]. 


\section{Stunned myocardium.}
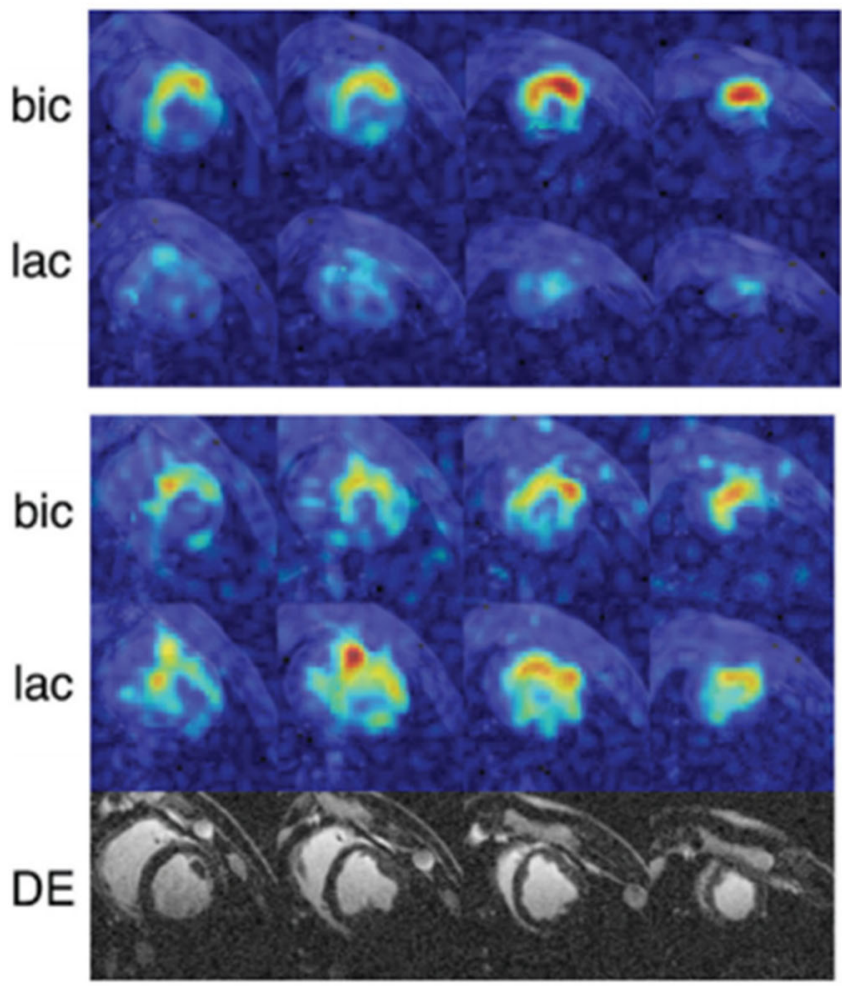

Infarcted myocardium.

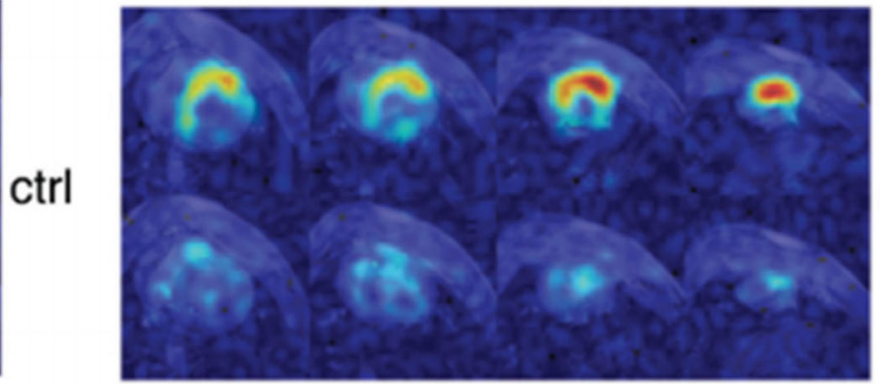

1 wk

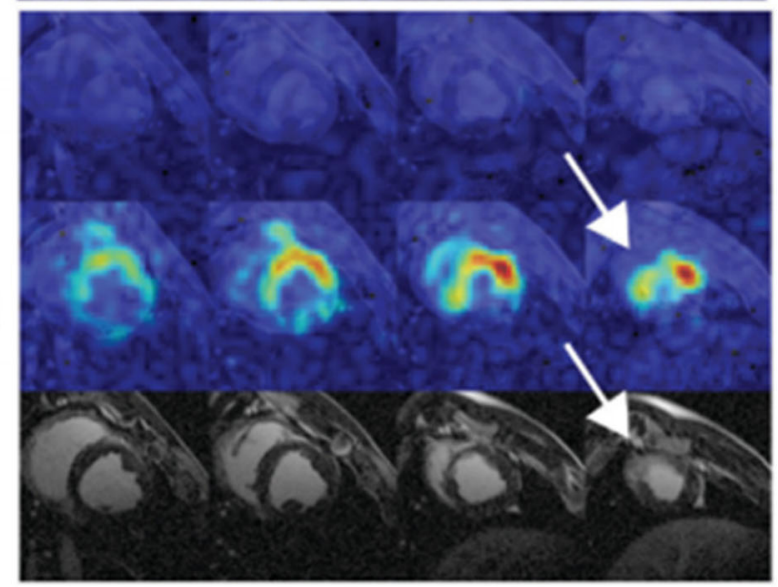

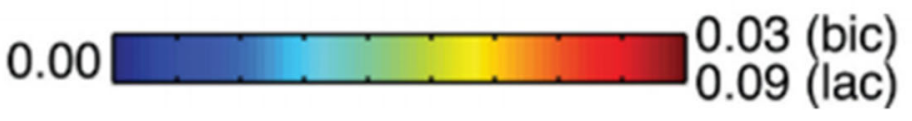

Fig. 3 Short axis imaging after 60-min LAD occlusion in pigs, at baseline and 1 week post-reperfusion. Color intensity is normalized to pyruvate seen in the LV cavity. Stunned myocardium (left) demonstrated normalization of bicarbonate (bic), absence of delayed enhancement (DE), and normalization of function at 1 week. In the MI (right),

\section{Myocardial Perfusion}

Myocardial perfusion imaging with SPECT or PET enables evaluation of location, extent, severity, and reversibility of myocardial perfusion defects in patients with known or suspected coronary artery disease (CAD), contributing to the detection of ischemic etiology of heart failure [81]. In addition to the assessment of relative distribution to the perfusion, PET with radiotracer kinetic modeling can be used to quantify myocardial blood flow (MBF) in absolute terms $(\mathrm{mL} / \mathrm{g} / \mathrm{min})$ at rest and during vasodilator stress that allows the computation of coronary flow reserve (CFR) [81]. Quantification of regional MBF and CFR by PET may identify microvascular dysfunction, better characterize the extent and severity of CAD in multi-vessel disease, detect balanced decreases of MBF in all major coronary artery vascular territories, and provide prognostic information beyond regional myocardial ischemia [82, 83]. Reduced CFR is a typical feature of a cardiomyopathic heart as a consequence of microvascular dysfunction even in the absence of epicardial bicarbonate production remained absent at 1 week; lactate (lac) was only seen in the peri-infarct region (arrow) and delayed enhancement clearly delineates the MI (adapted with permission from John Wiley and Sons: Lau AZ) [62]

CAD [84, 85]. Outcome studies have supported microvascular dysfunction as an independent contributing factor to the symptoms and progression of heart failure and reduced CFR was a predictor of adverse cardiac events in ischemic and dilated cardiomyopathy $[84,85]$.

\section{Myocardial Viability}

Myocardial viability and scarring can be assessed using perfusion imaging using specific viability protocols. In addition, 18F-fluorodeoxyglucose (18F-FDG) PET can be used to detect ischemic myocardium that is dysfunctional, but viable and has potential for recovery of the contractile function after revascularization [86]. Viable myocardium shows preserved 18F-FDG uptake, whereas markedly reduced or absent uptake indicates the presence of scar. A preserved or increased uptake of $18 \mathrm{~F}-\mathrm{FDG}$ in the presence of reduced myocardial perfusion, known as flow-metabolism mismatch, is the most commonly used marker of hibernating myocardium that is capable of 
functional recovery after revascularization (Fig. 4). 18F-FDG PET is a sensitive technique to detect viability and it predicts functional recovery upon revascularization. A pooled analysis of 24 studies in 756 patients demonstrated a weighed mean sensitivity and specificity of $92 \%$ and $63 \%$, respectively, for the detection of regional functional recovery [86]. Retrospective studies have also indicated lower annualized mortality rates of those with viable myocardium who underwent revascularization (4\%) versus those with viability who did not undergo revascularization (17\%) [88].

The value of 18F-FDG PET in guiding decisions on revascularization assigned 430 heart failure patients with an ejection fraction below $35 \%$ to either management assisted by $18 \mathrm{~F}-\mathrm{FDG}$ PET imaging or standard care [89]. Although the study overall showed only a nonsignificant trend toward reduction in cardiac events for 18F-FDG PET assisted management, 18F-FDG PET

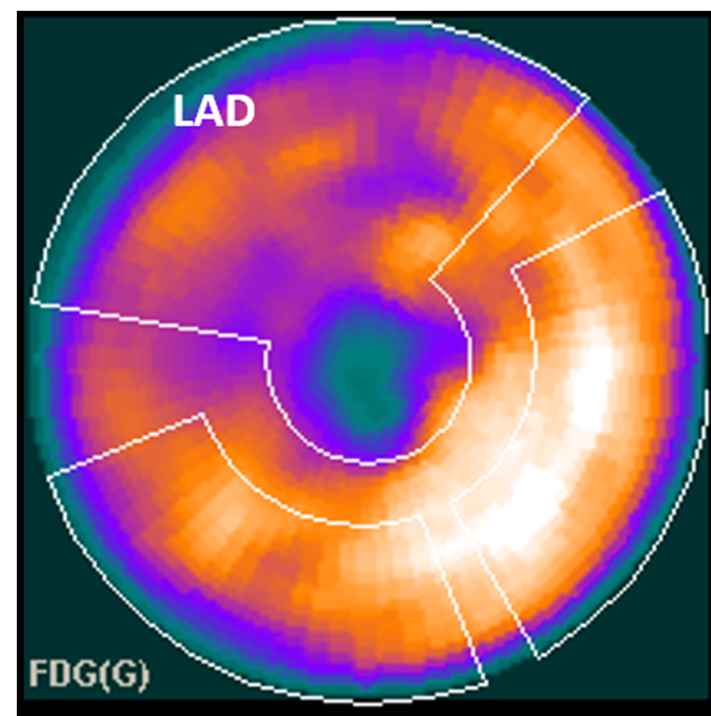

18F-FDG

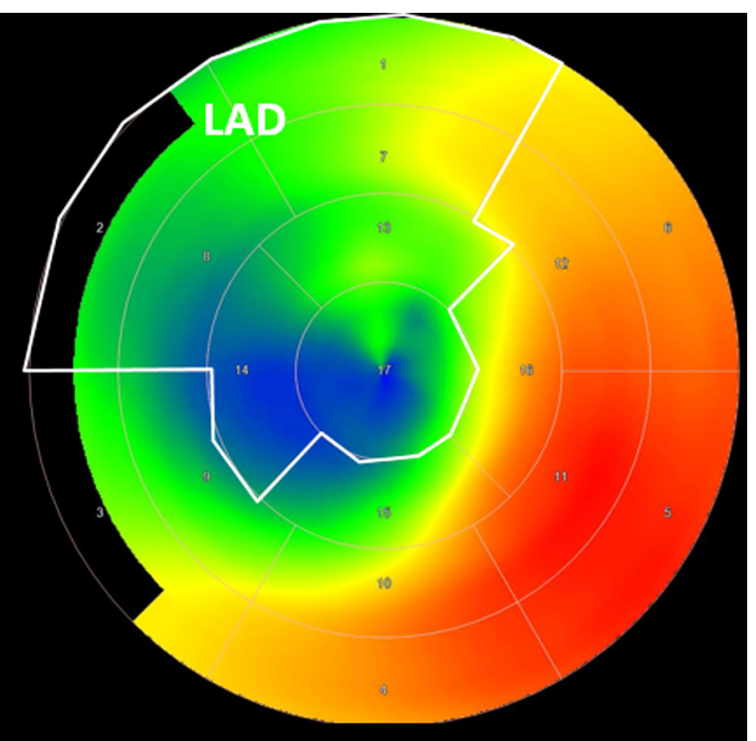

0-15-water perfusion

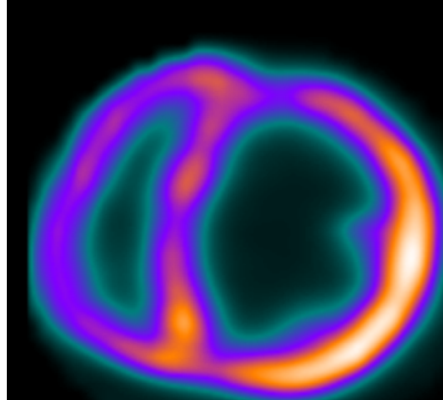

SA basal
SA mid
SA apex

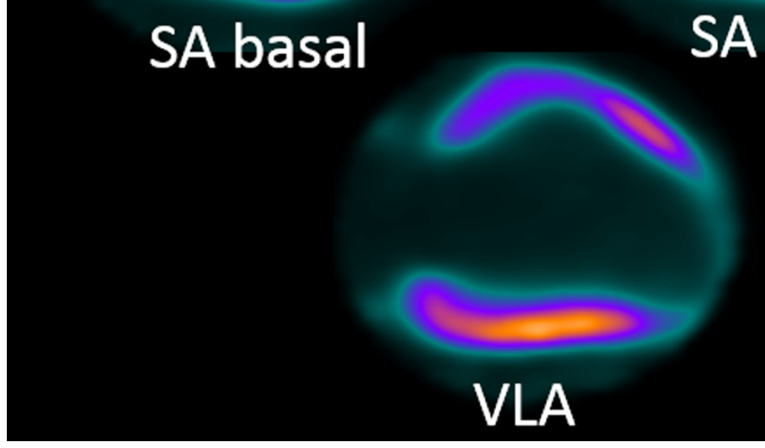

Fig. 4 Myocardial viability study using 18F-fluorodeoxyglucose (18FFDG) and myocardial perfusion study at rest using O-15-water. The patient had 3-vessel obstructive coronary artery disease, contractile dysfunction in the territory of the left anterior descending (LAD) coronary artery, reduced left ventricle ejection fraction (35\%), and high surgical risk. Resting perfusion is reduced in the LAD territory (white line). Viability study shows absence of $18 \mathrm{~F}-\mathrm{FDG}$ uptake in the apex, but partially preserved uptake elsewhere in the LAD territory indicating partially preserved viability. VLA vertical long axis, HLA horizontal long axis, SA short axis (adapted with permission from Springer Nature: Kiugel M) [87] 
assisted management improved outcomes in the subgroup of patients whose treatment adhered to the recommendations by imaging $[89,90]$, especially in patients with a large amount of hibernating myocardium [91]. Similarly, an observational study evaluating survival benefit from revascularization according to the extent of ischemic, scarred, and viable myocardium found survival benefit from revascularization in patients with hibernating myocardium $>10 \%$ of the left ventricle [92]. Current guidelines recommend that myocardial revascularization should be considered in patients with chronic ischemic heart failure with ejection fraction $\leq 35 \%$ in the presence of viable myocardium [93].

\section{Myocardial Metabolism}

In addition to $18 \mathrm{~F}-\mathrm{FDG}$, there are other PET tracers for the assessment of different aspects of myocardial metabolism [94, 95]. 11C-labeld acetate (11C-acetate) allows robust noninvasive measurement of myocardial oxygen consumption in the left and right ventricles independently of the substrate utilization [94]. This provides the means to estimate the oxygen cost of contractility, the efficiency of myocardial forward work. The finding of decreased efficiency of myocardial forward work is a consistent and early finding in cardiomyopathy caused by different etiologies [94]. Myocardial substrate metabolism can be studied in detail by fatty acid analogs, such as 18F-fluoro-6-thia-heptadecanoicacid or 11C-palmitate. The former reflects myocardial fatty acid utilization, whereas the latter reflects the flux of fatty acid metabolism through the cell including lipid pool storage, beta-oxidation, and tricarboxylic acid cycle [96]. Imaging of myocardial metabolism with PET has been used for evaluation of many medical and device therapies on the metabolism of the failing heart [94, 95].

\section{Cardiac Sympathetic Innervation}

Cardiac sympathetic imaging provides a non-invasive approach to assess alterations in cardiac sympathetic nerve function in cardiomyopathies [96, 97]. Heart failure is associated with an increased sympathetic tome characterized by increased release and decreased reuptake of norepinephrine by cardiac sympathetic nerve endings. Currently, cardiac sympathetic function is most commonly evaluated by SPECT imaging with 123Imetaiodobenzylguanidine (123I-MIBG), an iodinated neurotransmitter analog [97]. Uptake of 123I-MIBG in the heart is primarily mediated by the norepinephrine transporter (NET), an energy-dependent uptake mechanism. Cardiac uptake is usually measured relative to background mediastinal activity in planar images (heart-to-mediastinum ratio). Many studies have demonstrated that cardiac uptake of 123I-MIBG is reduced in individuals with heart failure and indicate that 123I-MIBG can be used as an independent predictor of heart failure progression and cardiac mortality [96-99].
11C-Metahydroxyephedrine (11C-HED) is a NET ligand that has been used for PET imaging of cardiac sympathetic function. Compared with SPECT, PET imaging provides a quantitative measure of sympathetic nerve function and identifies regional heterogeneity of innervation. The extent of reduced neural activity assessed with quantitative 11C-HED PET has been shown to be a predictor of sudden cardiac arrest independently of EF, infarct volume, symptoms, and natriuretic peptide levels in CAD patients who were candidates for an implantable cardioverter defibrillator placement for primary prevention of sudden cardiac death [100]. Despite benefits in terms of quantification and assessment of regional distribution, widespread clinical imaging with 11C-HED has been limited by the short radioactive half-life $(20 \mathrm{~min})$ and the need for an onsite cyclotron. N-[3-Bromo-4-(3-18F-fluoropropoxy)-benzyl]-guanidine (LMI1195) is a novel PET tracer that has been recently evaluated for evaluation of cardiac sympathetic neuronal function in man [101, 102]. Like MIBG, this agent is a benzylguanidine analog, but labeled with $18 \mathrm{~F}$. $18 \mathrm{~F}$ has a radioactive half-life of $120 \mathrm{~min}$ that would allow distribution to sites without an onsite cyclotron.

\section{New Tracers for MI and Remodeling}

New PET and SPECT tracers targeting the molecular mechanisms underlying repair of myocardial injury have been studied as potential markers of functional outcome after an acute MI [103]. Molecular imaging of the cellular mechanisms of myocardial remodeling can potentially provide new biomarkers for early detection, risk stratification, and evaluation of response to therapy in heart failure.

The $\alpha v \beta 3$ integrin is a mediator of angiogenesis and its expression is markedly upregulated in the myocardium after MI $[104,105]$. In addition to the endothelium, it is expressed by both activated cardiac myofibroblasts and macrophages after MI [105, 106]. Thus, $\alpha v \beta 3$ integrin has been studied as a potential target for imaging angiogenesis and repair of myocardial injury. Molecular imaging of $\alpha \mathrm{v} \beta 3$ is based on tracers that contain the RGD peptide subunit (the arginine-glycine-aspartate motif) that binds to the activated $\alpha v \beta 3$ integrin. Several PET tracers targeting $\alpha v \beta 3$ integrin have been evaluated in experimental models of MI [104, 106-113] and in patients with MI [114-116]. Studies have shown increased uptake of RGDbased radiotracers at the site of infarction as early as 3 days, peaking at 1-3 weeks after MI. The uptake correlates with angiogenesis, infarct scar formation, and adverse remodeling (Fig. 5). The value of imaging of $\alpha \mathrm{v} \beta 3$ integrin in predicting outcome of infarcted tissue after MI and demonstrating effects of therapies aimed at accelerating repair after MI, such as angiogenic gene therapy [117], still remains to be studied.

Inflammatory response after MI is another target that has been studied for predicting functional recovery after MI. Studies have shown increased myocardial uptake of metabolic 
markers 18F-FDG [118] and 11C-methionine [119] reflecting inflammatory activity after recent MI. Uptake of $18 \mathrm{~F}-\mathrm{FDG}$ early after an acute MI inversely correlated with the degree of functional recovery [119]. Pentixafor is a novel 68Ga-labeled PET tracer that binds to CXCR4 chemokine receptor mediating leukocytes accumulation at the sites of inflammation [120-122]. In experimental and human MI, increased pentixafor uptake was detected by PET in the infarcted tissue early after injury. After the initial pro-inflammatory phase, cells that promote tissue repair are the major inflammatory cell population in the infarcted myocardium [123, 124]. Molecular imaging may help to understand time course and contributions of the pro- and antiinflammatory mechanisms after MI.

Other radionuclide imaging approaches have been evaluated to assess molecular mechanisms underlying myocardial fibrosis, such as activation of matrix metalloproteinases [125-127] and activation of the renin-angiotensinaldosterone system [128, 129]. Molecular imaging with a radiolabeled ligand of the angiotensin receptor 1, 11CKR31173, demonstrated changes in myocardial expression

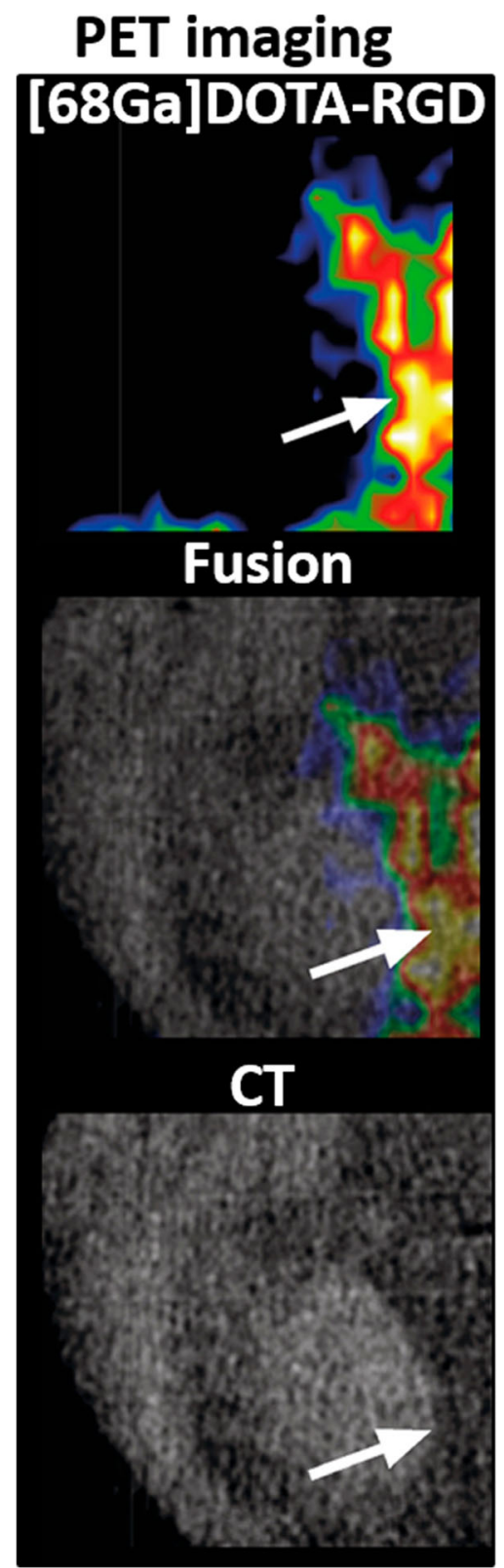

Fig. 5 Images of myocardial $\alpha v \beta 3$ integrin upregulation evaluated by 68Ga-DOTA-RGD PET after experimental myocardial infarction (MI) in rat. Autoradiographs of cross sections of the left ventricle show increased tracer uptake (green and red color) in the infarcted myocardium peaking at 1 week post-MI persisting at 4 weeks post-MI. Infarction is visible in

\section{Autoradiography}

HE

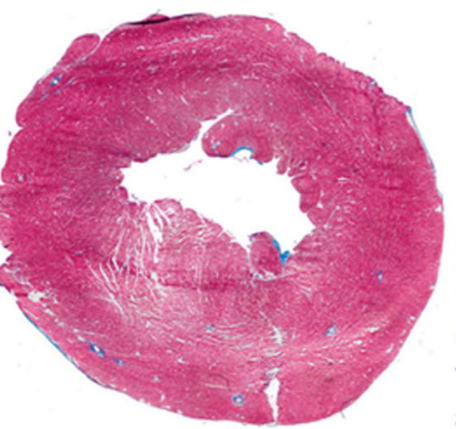

\section{Sham operation}

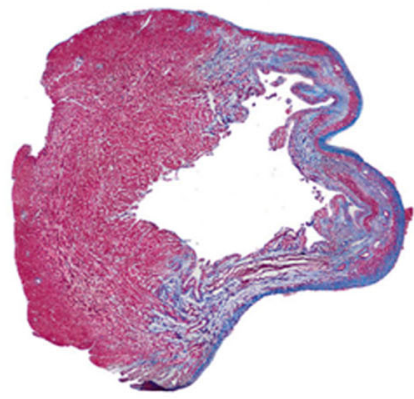

1 week post-MI

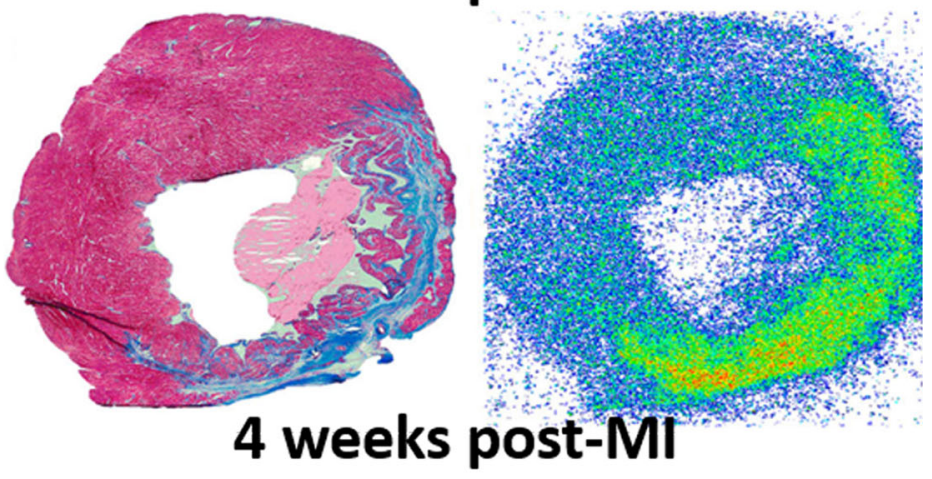

the corresponding section stained with hematoxylin and eosin (HE). Micro-PET/CT images show increased tracer uptake in the anterolateral wall of the left ventricle (arrow) 1 week after infarction (adapted with permission from Springer Nature: Kiugel M) [87] 
of angiotensin receptor 1 in a pig model of chronic MI and the radiotracer was tolerated also in humans [128]. Although new tracers for imaging MI are in a relatively early stage of development, studies have already shown that molecular imaging of the new targets can clarify pathogenesis of heart failure and be potentially useful to study effects of therapies.

\section{Conclusion}

Anatomy, function, and viability of the myocardium can be assessed by modern medical imaging techniques by both visualizing and quantifying damages. Novel imaging techniques are capable of precise and accurate visualization of the myocardium and detection of alternations at molecular level. Magnetic resonance imaging assesses the myocardium non-invasively with high spatial resolution and high contrast between myocardium and blood, cardiac function and tissue characterization of the myocardium. Molecular and metabolic conditions can also be assessed non-invasively with novel hyperpolarized magnetic resonance imaging. Single photon-emission tomography and positron-emission tomography are the most sensitive techniques to detect biological processes, including cardiac perfusion, metabolism, and viability in the myocardium.

Acknowledgments This study has been supported by the Finnish Academy Center of Excellence, Instrumentarium Science Foundation, Finnish Foundation for Cardiovascular Research, and Finnish Heart Foundation.

Funding Information Open access funding provided by University of Eastern Finland (UEF) including Kuopio University Hospital.

\section{Compliance with Ethical Standards}

Conflict of Interest Elias Ylä-Herttuala, Antti Saraste, Juhani Knuuti, Timo Liimatainen, and Seppo Ylä-Herttualaldeclare that they have no conflict of interest.

Human and Animal Rights and Informed Consent This article does not contain any studies with human or animal subjects performed by any of the authors.

Publisher's Note Springer Nature remains neutral with regard to jurisdictional claims in published maps and institutional affiliations.

\section{References}

Papers of particular interest, published recently, have been highlighted as:

- Of importance

- Of major importance

1. Yla-Herttuala S. Angiogennic gene therapy in cardiovascular diseases: dream or vision? Eur Heart J. 2017;38:1365-71.
2. Yla-Herttuala S. Cardiovascular gene therapy: past, present, and future. Mol Ther. 2017;25:1095-106.

3. Curley D. Molecular imaging of cardiac remodeling after myocardial infarction. Basic Res Cardiol. 2018;113:10.

4. van Slochteren FJ. Advanced measurement techniques of regional myocardial function to assess the effects of cardiac regenerative therapy in different models of ischemic cardiomyopathy. Eur Heart J Cardiovasc Imaging. 2012;13:808-18.

5. Patel N. Contrast - in cardiac magnetic resonance imaging. Echocardiography. 2018;35:401-9.

6. Mavrogeni S. T1 and T2 mapping in cardiology: "mapping the obscure object of desire". Cardiology. 2017;138:207-17.

7.• Yla-Herttuala E. Quantification of myocardial infarct area based on $\mathrm{T}_{\mathrm{RAFFn}}$ relaxation time maps - comparison with cardiovascular magnetic resonance late gadolinium enhancement, $T_{1 \rho}$ and $T_{2}$ in vivo. J Cardiovasc Magn Reson. 2018;20:34. This study provides the first myocardial infarction study in vivo done with $T_{\text {RAFFn }}$ relaxation time maps and those results are compared to other MRI imaging methods.

8. Ertl G. Healing after myocardial infarction. Cardiovasc Res. 2005;66:22-32.

9. Blankesteijn WM. Dynamics of cardiac wound healing following myocardial infarction: observations in genetically altered mice. Acta Physiol Scand. 2001;173:75-82.

10. Garg P. Role of T1 mapping and extracellular volume in the assessment of myocardial infarction. Anatol J Cardiol. 2018. https:// doi.org/10.14744/AnatolJCardiol.2018.39586.

11. Baxa J. T1 mapping of the ischemic myocardium: review of potential clinical use. Eur J Radiol. 2016;85:1322-928.

12. Galli A. Postinfarct left ventricular remodeling: a prevailing cause of heart failure. Cardiol Res Pract. 2016. https://doi.org/10.1155/ 2016/2579832.

13. Palazzuoli A. The impact if infarct size on regional and global left ventricular systolic function: a cardiac magnetic resonance imaging study. Int J Cardiovasc Imaging. 2015;5:1037-44.

14. van den Borne SW. Myocardial remodeling after infarction: the role of myofibroblasts. Nat Rev Cardiol. 2010;7:30-7.

15. Saeed M. Magnetic resonance imaging for characterizing myocardial diseases. Int J Cardiovasc Imaging. 2017;33:1395-414.

16. Captur G. Cardiac MRI evaluation of myocardial disease. Heart. 2016;102:1429-35.

17. Phelps ME. PET: the merging of biology and imaging into molecular imaging. J Nucl Med. 2000;41:661.

18. Apps A. Hyperpolarized magnetic resonance for in vivo real-time metabolic imaging. Heart. 2018;104:1484-91. This review article provides great overview about use of hyperpolarized MRI in vivo.

19. Ghosn MG. Important advances in technology and unique applications related to cardiac magnetic resonance imaging. Methodist Debakey Cardiovasc J. 2014;10:159-62.

20. Whitaker J. Cardiac MR characterization of left ventricular remodeling in a swine model of infarct followed by reperfusion. J Magn Reson Imaging. 2018;48:808-17. https://doi.org/10.1002/jmri. 26005 .

21. Krishnamurthy R. Tools for cardiovascular magnetic resonance. Cardiovasc Diagn Ther. 2014;4:104-25.

22. Wong DT. The role of cardiac magnetic resonance imaging following acute myocardial infarction. Eur Radiol. 2012;22:175768.

23. Reuben MT. Distal coronary embolization following acute myocardial infarction increases early infarct size and late left ventricular wall thinning in a porcine model. J Cardiovasc Magn Reson. 2015; 17:106

24. Watanabe E. Infarct tissue heterogeneity by contrast-enhanced magnetic resonance imaging is a novel predictor of mortality in 
patients with chronic coronary artery disease and left ventricular dysfunction. Circ Cardiovasc Imaging. 2014;7:887-94.

25. Pokorney SD. Infarct healing is a dynamic process following acute myocardial infarction. J Cardiovasc Magn Reson. 2012;14:62.

26. Michel L. Real-time pressure-volume analysis of acute myocardial infarction in mice. J Vis Exp. 2018. https://doi.org/10.3791/57621.

27. Opie LH. Controversies in ventricular remodeling. Lancet. 2006;367:356-67.

28. Larroza A. Texture analysis of cardiac cine magnetic resonance imaging to detect nonviable segments in patients with chronic myocardial infarction. Med Phys. 2018;4:1471-80.

29. Payne AR, Berry C, Kellman P, Anderson R, Hsu LY, Chen MY, et al. Bright-blood T(2)-weighted MRI has high diagnostic accuracy for myocardial hemorrhage in myocardial infarction: a preclinical validation study in swine. Circ Cardiovasc Imaging. 2011;4:738-45.

30. Kim HW. Dark-blood delayed enhancement cardiac magnetic resonance of myocardial infarction. JACC Cardiovasc Imaging. 2017;11:1758-69. https://doi.org/10.1016/j.jcmg.2017.09.021.

31. Caudron J. Evaluation of left ventricular diastolic function with cardiac MR imaging. Radiographics. 2011;31:239-59.

32. Shehata ML. Myocardial tissue tagging with cardiovascular magnetic resonance. J Cardiovasc Magn Reson. 2009;11:11-55.

33. Khan JN. Comparison of cardiovascular magnetic resonance feature tracking and tagging for the assessment of left ventricular systolic strain in acute myocardial infarction. Eur J Radiol. 2015;84:840-8.

34. Fahmy AS. Grey blood late gadolinium enhancement cardiovascular magnetic resonance for improved detection of myocardial scar. J Cardiovasc Magn Reson. 2018;20:20.

35. Maestrini V. T1 mapping for characterization of intracellular and extracellular myocardial diseases in heart failure. Curr Cardiovasc Imaging Rep. 2014;7:9287.

36. Lavin B. MRI with gadofosveset: a potential marker for permeability in myocvardial infarction. Atherosclerosis. 2018;275:400 8.

37. Kis E. Cardiac magnetic resonance imaging of the myocardium in chronic kidney disease. Kidney Blood Press Res. 2018;43:134 42.

38. Klein C. The influence of myocardial blood flow and volume of distribution on late gd-dtpa kinetics in ischemic heart failure. J Magn Reson Imaging. 2004;20:588-93.

39. Radenkovic D. T1 mapping in cardiac MRI. Heart Fail Rev. 2017;22:415-30.

40. Messroghli DR. Clinical recommendations for cardiovascular magnetic resonance mapping of $\mathrm{T} 1, \mathrm{~T} 2, \mathrm{~T} 2 *$ and extracellular volume: a consensus statement by the society for cardiovascular magnetic resonance (SCMR) endorsed by the European association for cardiovascular imaging (EACVI). J Cardiovasc Magn Reson. 2017;19:75.

41. Sibley CT. T1 mapping in cardiomyopathy at cardiac MR: comparison with endomyocardial biopsy. Radiology. 2012;265:724 32.

42. Mark Haacke E, et al. Magnetic resonance imaging, physical principles and sequence design. 1st ed. Hoboken: A John Wiley And Sons, Inc.; 1999.

43. Haaf P. Cardiac T1 mapping and extracellular volume (ECV) in clinical practice: a comprehensive review. J Cardiovasc Magn Reson. 2016;18:89.

44. Sanz J. Myocardial mapping with cardiac magnetic resonance: the diagnostic value of novel sequences. Rev Esp Cardiol (Eng Ed). 2016;69:849-61.

45. Aletras AH. Retrospective determination of the area at risk for reperfused acute myocardial infarction with $\mathrm{t} 2$-weighted cardiac magnetic resonance imaging: histopathological and displacement encoding with stimulated echoes (dense) functional validations. Circulation. 2006;113:1865-70.

46. Lota AS. T2 mapping and T2* imaging in heart failure. Heart Fail Rev. 2017;22:431-40.

47. Graham-Brown MP. Novel cardiac nuclear magnetic resonance method for noninvasive assessment of myocardial fibrosis in hemodialysis patients. Kidney Int. 2016;90:835-44.

48. Liu D. CMR native T1 mapping allows differentiation of reversible versus irreversible myocardial damage in ST-segment-elevation myocardial infarction. Circ Cardiovasc Imaging. 2017. https://doi.org/10.1161/CIRCIMAGING.116.005986.

49. Tessa C. T1 and T2 mapping in the identification of acute myocardial injury in patients with NSTEMI. Radiol Med. 2018;123: 926-34. https://doi.org/10.1007/s11547-018-0931-2.

50. Kali A. Determination of location, size, and transmurality of chronic myocardial infarction without exogenous contrast media by using cardiac magnetic resonance imaging at $3 \mathrm{~T}$. Circ Cardiovasc Imaging. 2014;7:471-81.

51. Stoffers RH. Assessment of myocardial injury after reperfused infarction by T1r cardiovascular magnetic resonance. J Cardiovasc Magn Reson. 2017;19:17.

52. Jackowski C. Postmortem unenhanced magnetic resonance imaging of myocardial infarction in correlation to histological infarction age characterization. Eur Heart J. 2006;27:2459-67.

53. Sepponen RE. A method for T1 rho imaging. J Comput Assist Tomogr. 1985;9:1007-11.

54. Mustafa HS. Longitudinal rotating frame relaxation time measurements in infarcted mouse myocardium in vivo. Magn Reson Med. 2013;69:1389-95.

55. van Oorschot JWM. Endogenous assessment of chronic myocardial infarction with T1 $\rho$-mapping in patients. J Cardiovasc Magn Reson. 2014;16:104-12.

56. Liimatainen T. MRI contrasts from relaxation along a fictitious field (RAFF). Magn Reson Med. 2010;64:983-94.

57.• Liimatainen T. MRI contrasts in high rank rotating frames. Magn Reson Med. 2015;73:254-62. This study provides the theoretical background behind the $\mathbf{T}_{\text {RAFFn }}$ relaxation time method.

58. Kettunen MI. Low spin-lock field $T_{1}$ relaxation in the rotating frame as a sensitive MR imaging marker for gene therapy treatment response in rat glioma. Radiology. 2007;243:796-803.

59. Khan MA. The follow-up of progressive hypertrophic cardiomyopathy using magnetic resonance rotating frame relaxation times. NMR Biomed. 2018;31. https://doi.org/10.1002/nbm.3871.

60. Rider OJ. Clinical implications of cardiac hyperpolarized magnetic resonance imaging. J Cardiovasc Magn Reson. 2013;15:93.

61. Golman K. Cardiac metabolism measured noninvasively by hyperpolarized 13C MRI. Magn Reson Med. 2008;59:1005-13.

62. Lau AZ. Reproducibility study for free-breathing measurements of pyruvate metabolism using hyperpolarized (13) $\mathrm{C}$ in the heart. Magn Reson Med. 2013;69:1063-71.

63. Merritt ME. Hyperpolarized $13 \mathrm{C}$ allows a direct measure of flux through a single enzyme-catalyzed step by NMR. Proc Natl Acad Sci U S A. 2007;104:19773-7.

64. Aquaro GD. Cardiac metabolism in a pig model of ischemiareperfusion by cardiac magnetic resonance with hyperpolarized 13C-Pyruvate. IJC Metab Endocr. 2015;6:17-23.

65. Ball DR. Metabolic imaging of acute and chronic infarction in the perfused rat heart using hyperpolarised [1-13C]pyruvate. NMR Biomed. 2013;26:1441-50.

66. Oh-Ici D. Hyperpolarized metabolic MR imaging of acute myocardial changes and recovery after ischemia-reperfusion in a small-animal model. Radiology. 2016;278:742-51.

67. Schroeder MA. Measuring intracellular $\mathrm{pH}$ in the heart using hyperpolarized carbon dioxide and bicarbonate: a $13 \mathrm{C}$ and $31 \mathrm{P}$ magnetic resonance spectroscopy study. Cardiovasc Res. 2010;86:82-91. 
68. Lau AZ. Mapping of intracellular $\mathrm{pH}$ in the in vivo rodent heart using hyperpolarized [1-13C]pyruvate. Magn Reson Med. 2017;77:1810-7.

69. Rubler S. New type of cardiomyopathy associated with diabetic glomerulosclerosis. Am J Cardiol. 1972;30:595-602.

70. Chong C-R. Metabolic remodelling in diabetic cardiomyopathy. Cardiovasc Res. 2017;113:422-30.

71. Do HP. Non-contrast assessment of microvascular integrity using arterial spin labeled cardiovascular magnetic resonance in a porcine model of acute myocardial infarction. J Cardiovasc Magn Reson. 2018;20:45.

72. Ma H. Contrast-enhanced whole-heart coronary MRA at 3.0T for the evaluation of cardiac venous anatomy. Int J Cardiovasc Imaging. 2011;27:1003-9.

73. Nquyen C. In vivo contrast free chronic myocardial infarction characterization using diffusion-weighted cardiovascular magnetic resonance. J Cardiovasc Magn Reson. 2014;16:68.

74. Sosnovik DE. Diffusion spectrum MRI tractography reveals the presence of a complex network of residual myofibers in infarcted myocardium. Circ Cardiovasc Imaging. 2009;2:206-12.

75. Payne AR. Bright-blood T2-weighted MRI has higher diagnostic accuracy than dark-blood short tau inversion recovery MRI for detection of acute myocardial infarction and for assessment of the ischemic area at risk and myocardial salvage. Circ Cardiovasc Imaging. 2011;4:210-9.

76. Arunachalam SP. Regional assessment of in vivo myocardial stiffness using 3D magnetic resonance elastography in a porcine model of myocardial infarction. Magn Reson Med. 2018;79:361-9.

77. Metha NK. Utility of cardiac magnetic resonance for evaluation of mitral regurgitation prior to mitral valve surgery. J Thorac Dis. 2017;4:S246-56.

78. Quarto C. Late gadolinium enhancement as a potential marker of increased perioperative risk in aortic valve replacement. Interact Cardiovasc Thorac Surg. 2012;15:45-50.

79. Bengel FM. Cardiac positron emission tomography. J Am Coll Cardiol. 2009;54:1-15.

80. Gaemperli O. Cardiac hybrid imaging. Eur Heart J Cardiovasc Imaging. 2012;13:51-60.

81. Jaarsma C. Diagnostic performance of noninvasive myocardial perfusion imaging using single-photon emission computed tomography, cardiac magnetic resonance, and positron emission tomography imaging for the detection of obstructive coronary artery disease: a meta-analysis. J Am Coll Cardiol. 2012;59:1719-28.

82. Saraste A. PET: is myocardial flow quantification a clinical reality? J Nucl Cardiol. 2012;19:1044-59.

83. Gupta A. Integrated noninvasive physiological assessment of coronary circulatory function and impact on cardiovascular mortality in patients with stable coronary artery disease. Circulation. 2017;136:2325-36.

84. Neglia D. Prognostic role of myocardial blood flow impairment in idiopathic left ventricular dysfunction. Circulation. 2002;105: 186-93.

85. Majmudar MD. Quantification of coronary flow reserve in patients with ischaemic and non-ischaemic cardiomyopathy and its association with clinical outcomes. Eur Heart J Cardiovasc Imaging. 2015;16:900-9.

86. Schinkel AF. Hibernating myocardium: diagnosis and patient outcomes. Curr Probl Cardiol. 2007;32:375-410.

87. Kiugel M. Dimeric [(68)Ga]DOTA-RGD peptide targeting $\alpha \mathrm{v} \beta 3$ integrin reveals extracellular matrix alterations after myocardial infarction. Mol Imaging Biol. 2014;16:793-801.

88. Allman KC. Myocardial viability testing and impact of revascularization on prognosis in patients with coronary artery disease and left ventricular dysfunction: a meta-analysis. J Am Coll Cardiol. 2002;39:1151-8.
89. Beanlands RS. F-18-Fluorodeoxyglucose positron emission tomography imaging-assisted management of patients with severe left ventricular dysfunction and suspected coronary disease a randomized, controlled trial (PARR-2). J Am Coll Cardiol. 2007;50: 2002-12.

90. Mc Ardle B. Long-term follow-up of outcomes with F-18fluorodeoxyglucose positron emission tomography imagingassisted management of patients with severe left ventricular dysfunction secondary to coronary disease. Circ Cardiovasc Imaging 2016;9(9). https://doi.org/10.1161/CIRCIMAGING.115.004331.

91. Mielniczuk LM. Does imaging-guided selection of patients with ischemic heart failure for high risk revascularization improve identification of those with the highest clinical benefit? Imagingguided selection of patients with ischemic heart failure for highrisk revascularization improves identification of those with the highest clinical benefit. Circ Cardiovasc Imaging. 2012;5:262-70.

92. Ling LF. Identification of therapeutic benefit from revascularization in patients with left ventricular systolic dysfunction: inducible ischemia versus hibernating myocardium. Circ Cardiovasc Imaging. 2013;6:363-72.

93. Ponikowski P. ESC guidelines for the diagnosis and treatment of acute and chronic heart failure: the task force for the diagnosis and treatment of acute and chronic heart failure of the European Society of Cardiology (ESC). Eur Heart J. 2016;37:2129-200.

94. Knaapen P. Myocardial energetics and efficiency: current status of the noninvasive approach. Circulation. 2007;115:918-27.

95. Tuunanen H, Knuuti J. Metabolic remodelling in human heart failure. Cardiovasc Res. 2011;90:251-7.

96. Juneau D. The role of nuclear cardiac imaging in risk stratification of sudden cardiac death. J Nucl Cardiol. 2016;23:1380-98.

97. Travin MI. Current clinical applications and next steps for cardiac innervation imaging. Curr Cardiol Rep. 2017;19:1.

98. Jacobson AF. Myocardial iodine-123 meta-iodobenzylguanidine imaging and cardiac events in heart failure. Results of the prospective ADMIRE-HF (AdreView Myocardial Imaging for Risk Evaluation in Heart Failure) study. J Am Coll Cardiol. 2010;55: 2212-21.

99. Narula J. ${ }^{123}$ I-MIBG imaging for prediction of mortality and potentially fatal events in heart failure: the ADMIRE-HFX study. $\mathrm{J}$ Nucl Med. 2015;56:1011-8.

100. Fallavollita JA. Regional myocardial sympathetic denervation predicts the risk of sudden cardiac arrest in ischemic cardiomyopathy. J Am Coll Cardiol. 2014;63:141-9.

101. Yu M, Bozek J. Evaluation of LMI1195, a novel 18F-labeled cardiac neuronal PET imaging agent, in cells and animal models. Circ Cardiovasc Imaging. 2011;4:435-43.

102. Sinusas AJ. Biodistribution and radiation dosimetry of LMI1195: first-in-human study of a novel 18F-labeled tracer for imaging myocardial innervation. J Nucl Med. 2014;55:1445-51.

103. Saraste A. PET imaging in heart failure: the role of new tracers. Heart Fail Rev. 2017;22:501-11.

104. Meoli DF. Noninvasive imaging of myocardial angiogenesis following experimental myocardial infarction. J Clin Invest. 2004;113:1684-91.

105. Sun M, Opavsky MA. Temporal response and localization of integrins beta 1 and beta 3 in the heart after myocardial infarction: regulation by cytokines. Circulation. 2003;107:1046-52.

106. Van den Borne SWM. Molecular imaging of interstitial alterations in remodeling myocardium after myocardial infarction. J Am Coll Cardiol. 2008;52:2017-28.

107. Higuchi T. Assessment of alphavbeta3 integrin expression after myocardial infarction by positron emission tomography. Cardiovasc Res. 2008;78:395-403.

108. Sherif HM. Molecular imaging of early $\alpha \mathrm{v} \beta 3$ integrin expression predicts long-term left-ventricle remodeling after myocardial infarction in rats. J Nucl Med. 2012;53:318-23. 
109. Gao H. PET imaging of angiogenesis after myocardial infarction/ reperfusion using a one-step labeled integrin-targeted tracer $18 \mathrm{~F}$ AlF-NOTA-PRGD2. Eur J Nucl Med Mol Imaging. 2012;39:68392.

110. Knetsch PA. [68Ga]NODAGA-RGD for imaging $\alpha \mathrm{v} \beta 3$ integrin expression. Eur J Nucl Med Mol Imaging. 2011;38:1303-12.

111. Laitinen I. Comparison of cyclic RGD peptides for $\alpha \mathrm{v} \beta 3$ integrin detection in a rat model of myocardial infarction. EJNMMI Res. 2013;3:38.

112. Menichetti L. MicroPET/CT imaging of $\alpha v \beta_{3}$ integrin via a novel 68Ga-NOTA-RGD peptidomimetic conjugate in rat myocardial infarction. Eur J Nucl Med Mol Imaging. 2013;40:1265-74.

113. Grönman M. Imaging of $\alpha v \beta 3$ integrin expression in experimental myocardial ischemia with [68Ga]NODAGA-RGD positron emission tomography. J Transl Med. 2017;15:144.

114. Jenkins WS. Cardiac $\alpha \mathrm{V} \beta 3$ integrin expression following acute myocardial infarction in humans. Heart. 2017;103:607-15.

115. Sun Y. Application of (68)Ga-PRGD2 PET/CT for $\alpha v \beta 3$-integrin imaging of myocardial infarction and stroke. Theranostics. 2014;4:778-86.

116. Verjans J. Early molecular imaging of interstitial changes in patients after myocardial infarction: comparison with delayed contrast-enhanced magnetic resonance imaging. J Nucl Cardiol. 2010;17:1065-72.

117. Hartikainen J. Adenoviral intramyocardial VEGF-D $\Delta \mathrm{N} \Delta \mathrm{C}$ gene transfer increases myocardial perfusion reserve in refractory angina patients: a phase I/Ila study with 1-year follow-up. Eur Heart J. 2017;38:2547-55.

118. Rischpler C. Prospective evaluation of $18 \mathrm{~F}$-fluorodeoxyglucose uptake in postischemic myocardium by simultaneous positron emission tomography/magnetic resonance imaging as a prognostic marker of functional outcome. Circ Cardiovasc Imaging. 2016;9: e004316.
119. Thackeray JT. Targeting amino acid metabolism for molecular imaging of inflammation early after myocardial infarction. Theranostics. 2016;6:1768-79.

120. Thackeray JT. Molecular imaging of the chemokine receptor CXCR4 after acute myocardial infarction. JACC Cardiovasc Imaging. 2015;8:1417-26.

121. Lapa C. [(68)Ga]Pentixafor-PET/CT for imaging of chemokine receptor 4 expression after myocardial infarction. JACC Cardiovasc Imaging. 2015;8:1466-8.

122. Rischpler C. Upregulated myocardial CXCR4-expression after myocardial infarction assessed by simultaneous GA- 68 pentixafor PET/MRI. J Nucl Cardiol. 2016;23:131-3.

123. Nahrendorf M. Monocyte and macrophage heterogeneity in the heart. Circ Res. 2013;112:1624-33.

124. Frangogiannis NG. The inflammatory response in myocardial injury, repair, and remodelling. Nat Rev Cardiol. 2014;11:255-65.

125. Sahul ZH. Targeted imaging of the spatial and temporal variation of matrix metalloproteinase activity in a porcine model of postinfarct remodeling: relationship to myocardial dysfunction. Circ Cardiovasc Imaging. 2011;4:381-91.

126. $\mathrm{Su} \mathrm{H}$. Noninvasive targeted imaging of matrix metalloproteinase activation in a murine model of postinfarction remodeling. Circulation. 2005;112:3157-67.

127. Kiugel M. Evaluation of $68 \mathrm{Ga}$-labeled peptide tracer for detection of gelatinase expression after myocardial infarction in rat. J Nucl Cardiol. 2018;25:1114-23.

128. Fukushima K. Molecular hybrid positron emission tomography/ computed tomography imaging of cardiac angiotensin II type 1 receptors. J Am Coll Cardiol. 2012;60:2527-34.

129. de Haas HJ. Molecular imaging of the cardiac extracellular matrix. Circ Res. 2014;114:903-15. 